\title{
Overutilization of bracing in the management of penetrating spinal cord injury from gunshot wounds
}

\author{
Nima Eftekhary, MD, ${ }^{1}$ Kenneth Nwosu, MD, ${ }^{2}$ Eric McCoy, MS,${ }^{3}$ Dudley Fukunaga, PA-C, ${ }^{4}$ and \\ Kevin Rolfe, MD, MPH ${ }^{2,4}$ \\ 1NYU Hospital for Joint Diseases, Department of Orthopaedic Surgery, New York, New York; ${ }^{2}$ Harbor-UCLA Medical Center, \\ Department of Orthopaedic Surgery, Torrance; ${ }^{3}$ David Geffen School of Medicine at UCLA, Office of Student Affairs, Los \\ Angeles; and ${ }^{4}$ Rancho Los Amigos Rehabilitation Center, Department of Spine Surgery, Torrance, California
}

OBJECTIVE Penetrating gunshot wounds (GSWs) to the spinal column are stable injuries and do not require spinal orthoses or bracing postinjury. Nonetheless, a high number of GSW-related spinal cord injury (SCI) patients are referred with a brace to national rehabilitation centers. Unnecessary bracing may encumber rehabilitation, create skin breakdown or pressure ulcers, and add excessive costs. The aim of this study was to confirm the stability of spinal column injuries from GSWs and quantify the overutilization rate of bracing based on long-term follow-up.

METHODS This retrospective cohort study was performed at a nationally renowned rehabilitation center. In total, 487 GSW-related SCI patients were transferred for rehabilitation and identified over the last 14 years. Retrospective chart review and telephone interviews were conducted to identify patients who were braced at the initial treating institution and determine if late instability, deformity, or neurological deterioration resulted in secondary surgery or intervention. In addition, 396 unoperated patients were available for analysis after 91 patients were excluded for undergoing an initial destabilizing surgical dissection or laminectomy, thereby altering the natural history of the injury. All of these 396 patients who presented with a brace had bracing discontinued upon reaching the facility.

RESULTS In total, 203 of 396 patients were transferred with a spinal brace, demonstrating an overutilization rate of $51 \%$. No patients deteriorated neurologically or needed later surgery for spinal column deformity or instability attributable to the injury. All patients had stable injuries. The patterns of injury and severity of neurological injury did not vary between patients who were initially braced or unbraced. The average follow-up was 7.8 years (range 1-14 years) and the average age was 25 years (range 10-62 years).

CONCLUSIONS The incidence of brace overutilization for penetrating GSW-related SCI was $51 \%$. Long-term follow-up in this study confirmed that these injuries were stable and thus did not require bracing. No patients deteriorated neurologically, whether or not they were initially braced. The unnecessary use of spinal orthoses increases costs and patient morbidity. Reeducation and dissemination of this information is warranted.

http://thejns.org/doi/abs/10.3171/2015.12.SPINE151022

KEY WORDS spine; spinal cord injury; bracing; cervical; thoracic; lumbar; gunshot wound; surgery; stability; instability; ASIA; penetrating trauma

$\mathrm{P}$ ENETRATING gunshot wounds (GSWs) to the spinal column are the second or third leading cause of spinal cord injury (SCI) in the United States. ${ }^{9}$ Unlike blunt trauma, spinal column injury from a penetrating GSW has little potential to create spinal column instability. Thus, the use of spinal bracing and orthoses appears unnecessary, with most cases of GSW-related spinal instability arising iatrogenically from surgical dissection or decompressive laminectomy. . $^{1,3,4,10,12,16,17}$

Despite these data, we continue to see a high number of GSW-related SCI patients referred to our national rehabilitation center with a brace from the outside treating facility. These braces interfere with rehabilitation efforts, create skin breakdown or pressure ulcers, and increase costs if they are not needed, ${ }^{2}$ and we universally discontinue bracing in these patients prior to engaging in a full rehabilitation program. As such, we sought to evaluate the difference between initially braced and unbraced patients in terms of the long-term sequelae of neurological decline or spinal deformity or instability requiring surgery.

ABBREVIATIONS ASIA = American Spinal Injury Association; $G S W=$ gunshot wound; $S C I$ = spinal cord injury.

SUBMITTED August 25, 2015. ACCEPTED December 7, 2015.

INCLUDE WHEN CITING Published online March 4, 2016; DOI: 10.3171/2015.12.SPINE151022. 


\section{Methods}

In total, 487 GSW-related SCI patients were transferred for rehabilitation to our institution and identified over a 14-year period (2000-2014). Ninety-one of these patients were excluded for having surgical procedures that were potentially destabilizing and prevented assessment of the true natural history of these injuries. A detailed retrospective review of the paper and electronic charts and radiological images of all participating patients was conducted by all authors. Telephone interviews were conducted to clarify missing or inadequate data in $13 \%$ of patients. Data were obtained for the included 396 unoperated patients with a follow-up period that was calculated based on the point of last clinical contact. Patients were referred from private, public, and academic hospital settings throughout the Los Angeles County basin and surrounding areas.

\section{Primary Study Goals}

First, all patients who were referred to our rehabilitation facility with a hard spinal brace or orthosis from an outside treating hospital were identified and compared with the patients who were referred without a brace in order to quantify the brace utilization rate.

Second, to judge the existing paradigm and determine that these injuries were stable and without the need for bracing (such that any bracing constitutes overbracing), we determined the number of subsequent or late surgeries that were performed for neurological deterioration or spinal instability, deformity, or imbalance across the available follow-up periods for each patient. The American Spinal Injury Association (ASIA) examination and ASIA impairment grade were used to assess neurological status or decline. Instability or deformity was declared if any late surgery for these sequelae was performed or if a treating surgical provider felt that surgery should be performed during follow-up based on the clinical or radiographic assessment, even if the surgery did not occur.

\section{Additional Study Goals}

Secondary factors that might influence the decision to brace, such as severity of the SCI or region of the spine involved (cervical, thoracic, or lumbosacral), were also analyzed.

All braced patients had bracing discontinued upon reaching our facility in order to allow the fullest participation in an organized rehabilitation program, which on average occurred less than 3 weeks after injury, and most patients had not been wearing the brace while in bed prior to transfer for active rehabilitation and mobilization. Also, only "hard" braces such as a thoracolumbosacral orthosis, Aspen, Philadelphia, or Miami J cervical hard collar (but not soft collar), cervicothoracic braces, lumbosacral orthosis, Minerva, sternal occipital mandibular immobilizer, and halo-vest or similar braces were considered as braces.

The statistical analysis was performed using the Statistical Package for the Social Sciences (IBM SPSS version $13)$, with $\mathrm{p}$ value significance chosen at the $\alpha=0.05$ level. Institutional review board approval for the study was obtained.

\section{Results}

In total, 203 of 396 patients were transferred with a spinal orthosis, demonstrating an actual utilization rate of $51 \%$. The average follow-up period was 7 years (range $1-14$ years), with an average age of 25 years (10-62 years) at the time of injury. In total, $2 \%$ of patients achieved only the 1-year minimum follow-up, $3 \%$ reached only 2 years, and all others achieved 3 or more years. Ninety-five percent of all injuries occurred in males. After universal discontinuation of bracing upon presentation at our facility, none of the patients, whether initially braced or not, have had a subsequent surgery for neurological deterioration or spinal decompensation related to instability, deformity, or imbalance that was attributable to the GSW-related SCI (Table 1), so bracing constituted overutilization in $51 \%$ of treated patients. Two patients did undergo subsequent surgery for other reasons. One patient with an L-4 incomplete neurological injury (ASIA Grade C), who was initially braced, underwent an instrumented L4-5 posterior fusion with a bone graft for pain due to a Charcot joint at 8 months postinjury. The pain predated the development of the Charcot joint and remained unrelieved and unchanged at 10 years of follow-up despite successful radiographic fusion. A second patient with a T-8 complete injury (ASIA Grade A), who was injured before puberty, developed postparalytic scoliosis attributable to the SCI. Among the 91 of 487 patients excluded for having an initial surgery, 12 (2.4\%) underwent surgery and fusion for presumed instability.

There were 280 patients with complete SCIs who were assigned to ASIA Grade A status and 116 patients with incomplete ASIA Grade B, C, or D status. Of 280 patients with complete injuries, 150 were braced (54\%). Of 116 patients with incomplete injuries, 53 were braced (46\%). There was no significant difference in the decision to brace based on the completeness of the neurological injury $(\mathrm{p}=0.15)$.

Bracing by spinal region included 63 of 103 cervical injuries (61\%), 109 of 229 thoracic injuries (48\%), and 31 of 64 lumbar injuries (48\%) (Table 2). There was a nonsignificant statistical trend toward increased brace utilization with cervical injuries ( $\mathrm{p}=0.06)$.

Brace utilization was statistically and significantly different for the earlier time period (49\% during 2000-2007) versus the later time period (61\% during 2008-2014) $(\mathrm{p}=$ 0.006).

\section{Discussion}

Among the patients with GSW-related SCI referred to our institution, $51 \%$ were unnecessarily braced. The longterm clinical and radiographic follow-up in this study, which averaged 7 years and was as long as 14 years in some patients, confirms that these injuries were stable and not in need of a brace, as none of the patients required subsequent surgery for neurological or spinal imbalance, deformity, or instability attributable to the GSW-related SCI. Two patients, however, did undergo subsequent surgery. One patient with an L-4 ASIA Grade C injury underwent surgery for pain due to a Charcot joint. The patient's pain, however, predated the development of the Charcot joint and did not change despite successful fusion. Thus, the 
TABLE 1. Overall rate of overbracing*

\begin{tabular}{lrrrc}
\hline \multicolumn{1}{c}{ Characteristic } & Brace & Brace & Total & $\%$ Braced \\
\hline Patients w/ GSW SCI & 203 & 193 & 396 & 51 \\
\hline $\begin{array}{l}\text { No. of late neurological deterio- } \\
\text { rations }\end{array}$ & 0 & 0 & 0 & 0 \\
\hline $\begin{array}{l}\text { No. of late surgeries for spinal } \\
\text { column instability or decom- } \\
\text { pensation }\end{array}$ & 0 & 0 & $0 \dagger$ & 0 \\
\hline
\end{tabular}

* Values are shown as number of patients or percentage.

$\dagger$ Two patients had late surgery for other reasons (see Results).

pain was likely neuropathic from the neurological injury and the Charcot joint an incidental occurrence, for which surgery was poorly indicated. The second patient was a prepubertal male who sustained a midthoracic ASIA Grade A neurological injury that developed into postparalytic neuromuscular-type scoliosis. The patient underwent a T-2 to pelvis fusion. The occurrence of scoliosis in growing children who sustain a severe SCI in the cervical or thoracic spine is well documented in the literature and occurs due to the SCI, regardless of the mechanism or stability of the SCI itself.6,11,15 As a result, we submit that neither of these 2 events constitutes true instability or deformity that arises from the GSW-related SCI. As such, no patient truly required subsequent surgery for spinal column instability, deformity, or decompensation, and none deteriorated neurologically. In addition, any bracing was universally discontinued (except for surgical exclusions that disrupted the natural history of the injury), further confirming the evidence for the paradigm that these injuries are stable and not in need of bracing. Among the patients excluded for surgery, only 12 of 91 surgeries were performed for presumed instability and $76 \%$ of the total were deemed unindicated upon review of the current literature standards and will be the topic of a separate report.

Most studies in the literature agree with our results that these injuries are stable. The largest of these studies found no spinal instability in a series of 1300 patients..$^{14}$ Another study showed no instability in 60 patients with penetrating SCI and bony involvement, despite 2 patients with what would have been Denis 2-column instability on imaging. ${ }^{4}$ One study noted that there have been no reported cases of thoracic instability after GSW-related column injury due to the inherent stability of the thoracic vertebrae created by the costovertebral joints and the ribs. ${ }^{17}$

Despite the apparent stability of these injuries, a number of studies have suggested spinal column instability following a GSW. A retrospective study of 37 patients with a GSW to the spine noted 3 patients with cervical injuries and 1 patient with lumbar injury that were deemed to be unstable. However, the blunt mechanism-derived Denis classification was used despite never having been validated for a penetrating mechanism. ${ }^{4}$ In another 2 studies, 4 of 65 patients with cervical spine fractures were labeled unstable and underwent surgery in the first study, as did 3 of 19 patients with cervical spine fractures in the second study. ${ }^{12,13}$ Again, this "instability" is without historical precedent in decompensation or controls.

Ultimately, it appears that various secondary rationales
TABLE 2. Overbracing by spinal level ${ }^{*}$

\begin{tabular}{lcccc}
\hline Spine Level & Brace & No Brace & Total & $\%$ Braced \\
\hline Cervical & 63 & 40 & 103 & $61 \dagger$ \\
\hline Thoracic & 109 & 120 & 229 & 48 \\
\hline Lumbar & 31 & 33 & 64 & 48 \\
\hline * Values are shown as number of patients or percentage. \\
$\begin{array}{l}\dagger \text { Statistically nonsignificant trend toward greater brace overuse in cervical } \\
\text { injuries }(p=0.065) .\end{array}$
\end{tabular}

for assuming the instability in GSW-related spinal injuries have been used and applied without a good understanding of the untreated natural history of these injuries. Declarations of instability in the literature have been made using items such as 1) the Denis 3-column model as applied to blunt injury mechanisms, 2) high- versus low-velocity missiles, 3) side-to-side bullet trajectories and fracture patterns, and 4) downward superior-to-inferior fracture patterns. ${ }^{5,7,8,13,17}$ Cervical injuries seem to instill greater concern for instability in the literature. We surmise that the trend we saw for increased brace use is due to the absence of ribs and the smaller size of the vertebrae in the cervical region. The assessment in the present study revealed no trend toward increased bracing based on the completeness of the SCI. While this and other studies have tried to pinpoint these secondary reasons as to why providers have used bracing, the present study suggests that bracing is unnecessary in the first place. This study does not support the use of any of these rationales, but rather demonstrates the natural history of these injuries as being stable over long-term follow-up. In our 396 patients, every combination of injury-from superior-to-inferior or side-to-side GSW-directed fracture patterns, Denis 2and 3-column patterns, and high-velocity military rifle injuries (especially from AK-47s, which have infiltrated among our Los Angeles street gangs) - were multiplied to represent each region of the spine (cervical, thoracic, and lumbar) and yet none deteriorated neurologically or required subsequent surgery for instability or decompensation. Evaluation of the excluded 91 surgically treated patients does not controvert these data and will be the subject of a separate report, as previously mentioned. It is significant to note that the SCI patterns were not more severe in those operated patients.

This broad statement of stability may not be without exception. A notable exception is the idea of bipedicular fractures or comminuted facet fractures. In this series, however, none of 3 bipedicular fractures and multiple comminuted facet fracture patients had late instability or required surgery. Thus, it is unclear if these exceptions apply at some level, but they did not in this series. Also, $88 \%$ (292 of 332 patients) of our cord-level injuries in the thoracic and cervical regions were complete or motor complete (ASIA Grade A or B) without developing future changes in ASIA grade. Such patients have little to lose through observation and are the most at risk for pressure ulcers caused by bracing. Our recommendation is that "no brace" should be the rule after GSW-related spinal injury unless there is clear neurological deterioration in a motorincomplete patient. 
Bracing is not without consequence, nor is it benign. It raises costs, impedes rehabilitation efforts (e.g., delays in mobilization or interference with mobilization efforts), and adds the risk of skin breakdown. Pressure-related skin breakdown, or pressure sores, are the most common complication of orthoses, especially cervical collars, and, as discussed previously, the cervical region was the most frequently braced. ${ }^{2}$ Spinal orthoses can also lead to decompensation and deconditioning of the paraspinal musculature and trunk stabilizers, predisposing the patient to increased pain while encumbering rehabilitation and ambulation. Dysphagia and alterations in swallowing have also been associated with cervical orthoses. ${ }^{2}$

The limitations of this study include its retrospective nature and any inaccuracies attributable to interpreting incorrect or incomplete content in medical records, charts, and radiological studies. Though patient telephone interviews were conducted to confirm unclear or undocumented data, patient recall bias may be present. It should be emphasized that longer or more exhaustive follow-up might reveal cases that have not been found or may yet surface. Also, an unknown radiological deformity, which was not bothersome or evident to a patient but which might be considered significant to the treating surgeon, may be present in patients with recent clinical follow-up but without corresponding radiographs at the point of last contact.

\section{Conclusions}

This large study confirms the paradigm that penetrating GSW-related spinal injuries are stable injuries that do not require bracing, which was further corroborated by long-term follow-up without subsequent neurological deterioration or spinal column instability that was attributed to the SCI. Nonetheless, bracing continues to be used by treatment providers in more than $50 \%$ of cases, adding costs, encumbrance to rehabilitation, and risk of skin breakdown with statistically significant increased use in recent years compared with prior years. Part of the brace overutilization problem may be that any one spinal surgeon sees relatively few of these penetrating injuries in his career and the injury patterns often appear unstable by traditional blunt-mechanism classification schemes. Reeducation and dissemination of this information to spinal physicians is warranted.

\section{References}

1. Aarabi B, Alibaii E, Taghipur M, Kamgarpur A: Comparative study of functional recovery for surgically explored and conservatively managed spinal cord missile injuries. Neurosurgery 39:1133-1140, 1996

2. Agabegi SS, Asghar FA, Herkowitz HN: Spinal orthoses. J Am Acad Orthop Surg 18:657-667, 2010

3. Amar AP, Levy ML: Surgical controversies in the management of spinal cord injury. J Am Coll Surg 188:550-566, 1999

4. Aryan HE, Amar AP, Ozgur BM, Levy ML: Gunshot wounds to the spine in adolescents. Neurosurgery 57:748752,2005
5. Bono CM, Heary RF: Gunshot wounds to the spine. Spine $\mathbf{J}$ 4:230-240, 2004

6. Dearolf WW III, Betz RR, Vogel LC, Levin J, Clancy M, Steel HH: Scoliosis in pediatric spinal cord-injured patients. J Pediatr Orthop 10:214-218, 1990

7. Denis F: The three column spine and its significance in the classification of acute thoracolumbar spinal injuries. Spine (Phila Pa 1976) 8:817-831, 1983

8. Duz B, Cansever T, Secer HI, Kahraman S, Daneyemez MK, Gonul E: Evaluation of spinal missile injuries with respect to bullet trajectory, surgical indications and timing of surgical intervention: a new guideline. Spine (Phila Pa 1976) 33:E746-E753, 2008

9. Isiklar ZU, Lindsey RW: Low-velocity civilian gunshot wounds of the spine. Orthopedics 20:967-972, 1997

10. Jallo GI: Neurosurgical management of penetrating spinal injury. Surg Neurol 47:328-330, 1997

11. Lancourt JE, Dickson JH, Carter RE: Paralytic spinal deformity following traumatic spinal-cord injury in children and adolescents. J Bone Joint Surg Am 63:47-53, 1981

12. Lustenberger T, Talving P, Lam L, Kobayashi L, Inaba K, Plurad D, et al: Unstable cervical spine fracture after penetrating neck injury: a rare entity in an analysis of $1,069 \mathrm{pa}-$ tients. J Trauma 70:870-872, 2011

13. Medzon R, Rothenhaus T, Bono CM, Grindlinger G, Rathlev NK: Stability of cervical spine fractures after gunshot wounds to the head and neck. Spine (Phila Pa 1976) 30:2274-2279, 2005

14. Meyer PR, Apple DF, Bohlman HH, Ferguson RL, Stauffer ES: Symposium: management of fractures of the thoracolumbar spine. Contemp Orthop 16:57-86, 1988

15. Mulcahey MJ, Gaughan JP, Betz RR, Samdani AF, Barakat N, Hunter LN: Neuromuscular scoliosis in children with spinal cord injury. Top Spinal Cord Inj Rehabil 19:96-103, 2013

16. Stauffer ES, Wood RW, Kelly EG: Gunshot wounds of the spine: the effects of laminectomy. J Bone Joint Surg Am 61:389-392, 1979

17. Yoshida GM, Garland D, Waters RL: Gunshot wounds to the spine. Orthop Clin North Am 26:109-116, 1995

\section{Disclosures}

The authors report no conflict of interest concerning the materials or methods used in this study or the findings specified in this paper.

\section{Author Contributions}

Conception and design: Eftekhary, Nwosu, Fukunaga, Rolfe. Acquisition of data: all authors. Analysis and interpretation of data: all authors. Drafting the article: Eftekhary, Nwosu, Rolfe. Critically revising the article: Eftekhary, Nwosu, Rolfe. Reviewed submitted version of manuscript: all authors. Approved the final version of the manuscript on behalf of all authors: Eftekhary. Statistical analysis: Eftekhary, McCoy, Rolfe. Administrative/technical/material support: Eftekhary, McCoy, Fukunaga, Rolfe. Study supervision: Rolfe.

\section{Correspondence}

Nima Eftekhary, Department of Orthopaedic Surgery, NYU Hospital for Joint Diseases, 545 First Ave., Apt. 8A, New York, NY 10016.email: nima.eftekhary@nyumc.org. 